\title{
Wave Function Is not Amplitude of Probability Density
}

\author{
Marat Vladimirovich Guryev \\ Independent Scholar, Moscow, Russian Federation
}

\section{Email address:}

mvguryev@mail.ru

\section{To cite this article:}

Marat Vladimirovich Guryev. Wave Function Is not Amplitude of Probability Density. American Journal of Modern Physics. Vol. 6, No. 4, 2017, pp. 49-50. doi: 10.11648/j.ajmp.20170604.11

Received: March 24, 2017; Accepted: April 25, 2017; Published: June 14, 2017

\begin{abstract}
Wave function (WF) is usually accepted to be the amplitude of probability density of a particle at a given point of phase space. This assertion is often referred to as Born postulate (BP) which is one of foundations of quantum mechanics. It is obvious that BP requires the normalization of WF to the number of indistinguishable particles in the object under study. But calculation methods of quantum mechanics require normalization to unit regardless of the number of electrons in the atom. The simplest way to solve this contradiction is to reject BP and acknowledge that normalization of WF has no relation to the number of particles in the object under consideration. This contradiction was not noticed until now because BP was tested for WF of single particles only.
\end{abstract}

Keywords: Wave Function, Probability Density, Born Postulate, Normalization

\section{Introduction}

In this article, we will consider the following well known assertion: value of wave function of (WF) of a particle at a given point of phase space represents the amplitude of probability density of the particle to be at this point, see e $g$ [1-7]. This assertion can be considered as definition of Born postulate (BP) which is usually considered as important part of the Copenhagen interpretation of quantum mechanics [8]. The most detailed description of properties of WF in terms of $\mathrm{BP}$ is given in review in [9].

The usefulness of BP is, at first glance, convincingly illustrated by numerous examples of probability density for solutions of Schrödinger or Dirac equations within atomic quantum electrodynamics (QED) [10].

However, BP has never been applied and carefully considered for many-electron atoms (and molecules). The aforementioned examples relate only to one-electron atoms or ions. We will see below that in this case important contradiction, revealed in this article, is hidden.

Therefore, we will mainly consider the use of BP for many-electron objects. We will consider only $N$-electron atoms, ions, and molecules where $N>1$.

The remainder of the paper is organized as follows: Section 2 contains proof of contradiction between BP and standard calculation methods of quantum mechanics.
Possible objections are considered in section 3. Short conclusion is given in section 4 .

\section{Contradiction Between BP and Methods of Quantum Mechanics}

Since the WF of an $N$-electron atom describes the motion of $N$ electrons, BP requires the expression $|\psi|^{2}$ to represent probability densities of $N$ indistinguishable electrons. On the surface, there are no problems, because standard equations of $\mathrm{QM}$ are homogeneous, and we always can normalize to $N$ the WF of any many-electron object.

Yet, in fact, there is a serious problem. We should recall that we achieve all the results of $\mathrm{QM}$ through the normalization of the complete WF of the bound state to unit. Continuum WF are usually normalized to $\delta$ - function, which reduces to unit after standard integration. The calculation methods of QM, e.g., the construction of Green's functions, also only need unit normalization. Calculation methods of QM require normalization to unit regardless of the number of electrons in the atom and the number of electrons and nucleuses in the molecule. Last, but not least, we know that all precise results of QM, including QED, are confirmed by all the experimental data. Therefore, we cannot normalize to $N$ the WF of $\mathrm{N}$-electron object while 
remaining within standard QM.

Revealed contradiction was not noticed until now because BP was tested for WF of single particles only. As a result, this contradiction was hidden all the time before this paper was written.

\section{Possible Objections}

Just in case, we should consider the possibility of "adventurous" explanations of this contradiction. First of all we should be sure that any atom or molecule, which we accept as an $\mathrm{N}$-electron one, actually does contain exactly $\mathrm{N}$ electrons. Two main examples follow, which support this statement.

First, we know that atomic mass $M_{a}=N m_{e}+M_{n}$, where $m_{e}$ and $M_{n}$ are electron and nucleus masses, respectively. This equality is validated by numerous precise experiments (e.g., [11] and references therein).

Second, Dirac's and Schrödinger's $N$-electron atomic Hamiltonians are exact sums of $N$ one-electron Hamiltonians, with additional $N(N-1) / 2$ terms describing electron-electron interaction (in addition to nuclear Coulomb potential). This implies that only $N$ electrons actually exist in the atom in our equations.

We must acknowledge that normalization of WF, which we use in real calculations in quantum mechanics, is not connected with the number of particles in the object. Therefore, we must conclude that BP really contradicts actual QM.

In spite of this, all real precise computational results within QM and QED for atoms and molecules coincide well with the results of corresponding measurements (e.g., [10] and references therein). This implies that BP actually is not in use in these calculations; otherwise, we would notice some contradictions of results of atomic QED calculations to precise experiments.

\section{Conclusion}

As the simplest step toward the solution of the raised problem, we can acknowledge that BP is merely an unfounded verbal interpretation of the quantity $|\psi|^{2}$ and nothing more. We have shown that BP is incompatible with real quantum mechanics. It follows from definition of BP that in the general case WF is not amplitude of probability density. It is obvious that we can manage with QED without BP. Yet, in this case, we cannot say today that BP is a part of QM. Now we should be more careful with verbal interpretation of quantum physics.

\section{References}

[1] J. L. Basdevant, and J. Dalibard, "Quantum Mechanics", Springer; Berlin, 2002.

[2] S. Gasiorowiczs, "Quantum Physics," third Edition, John Wiley \& Sons Ltd., New York, 2003.

[3] W. Greiner, "Quantum Mechanics, an Introduction," fourth Edition, Springer, Berlin, 2001.

[4] L. I. Schiff, "Quantum Mechanics," McGraw-Hill, New York, 1949.

[5] E. Merzbacher, "Quantum Mechanics", second Edition, John Wiley and Sons Ltd., New York, 1970.

[6] D. J. Griffiths, "Introduction to Quantum Mechanics," Prentice Hall, Upper Saddle River, 1995.

[7] F. Schwabl, "Quantum Mechanics", fourth Edition, Springer Berlin Heidelberg New York, 2007.

[8] D. Bohm, "Quantum Theory”, Prentice Hall, New York, 1952.

[9] W. H. Zurek, Probabilities from entanglement, Born's rule $p_{k}=\left|\psi_{k}\right|^{2}$ from envariance, Phys. Rev. A 71, 052105, (2005).

[10] Grant, I. P. "Relativistic Quantum Theory of Atoms and Molecules", Springer Science+Business Media; New York, 2007.

[11] Matthew Redshaw, B. Pianna, J. Mount and Edmund G. Myers, Penning-trap measurement of the atomic masses of ${ }^{18} \mathrm{O}$ and ${ }^{19} \mathrm{~F}$ uncertainties $<0.1$ parts per $10^{9}$; Phys. Rev. A 79, 012507 (2009). 